\title{
Restrictive mitral valve annuloplasty for chronic ischaemic mitral regurgitation: outcomes of flexible versus semi-rigid rings
}

\author{
Philip Y. K. Pang ${ }^{1}$, Ming Jie Huang1, Teing Ee Tan ${ }^{1}$, See Lim Lim ${ }^{1}$, Madhava J. Naik ${ }^{1}$, Victor T. T. Chao ${ }^{1}$, \\ Yoong Kong Sin ${ }^{1}$, Chong Hee Lim ${ }^{1,2}$, Yeow Leng Chua ${ }^{1}$ \\ ${ }^{1}$ Department of Cardiothoracic Surgery, National Heart Centre Singapore, Singapore; ${ }^{2} \mathrm{C}$ H Lim Thoracic Cardiovascular Surgery, Mount Elizabeth \\ Medical Centre, Singapore \\ Contributions: (I) Conception and design: PYK Pang, YL Chua; (II) Administrative support: PYK Pang, MJ Huang; (III) Provision of study materials or \\ patients: PYK Pang, TE Tan, SL Lim, MJ Naik, VTT Chao, CH Lim, YK Sin, YL Chua; (IV) Collection and assembly of data: PYK Pang, MJ Huang; \\ (V) Data analysis and interpretation: PYK Pang, MJ Huang; (VI) Manuscript writing: All authors; (VII) Final approval of manuscript: All authors. \\ Correspondence to: Philip Y. K. Pang. National Heart Centre Singapore, 5 Hospital Drive, Singapore 169609. Email: philip.pang.y.k@singhealth.com.sg.
}

\begin{abstract}
Background: Restrictive mitral annuloplasty is the mainstay of surgical correction of chronic ischaemic mitral regurgitation (CIMR). Long-term data on the various types of annuloplasty rings is limited. The aim of this study was to investigate the clinical and echocardiographic outcomes of restrictive mitral annuloplasty in patients with CIMR, comparing the use of flexible versus semi-rigid annuloplasty rings.

Methods: A retrospective review was conducted for 133 patients with CIMR who underwent restrictive mitral annuloplasty at our institution between 1999 and 2015. Patient demographics and postoperative outcomes were analyzed.
\end{abstract}

Results: Mean age was $61.9 \pm 9.2$ years and 103 patients $(77.4 \%)$ were male. All patients underwent coronary artery bypass grafting, with a mean of $3.3 \pm 0.8$ grafts. Flexible rings was implanted in 39 patients $(29.3 \%$, group F) and semi-rigid rings in 94 (70.7\%, group R). Preoperative New York Heart Association class was III/IV in 104 patients (78.2\%). Mean preoperative left ventricular ejection fraction was $28.8 \% \pm 10.2 \%$. Preoperative mitral regurgitation was moderate in 51 patients $(38.3 \%)$ and severe in 82 (61.7\%). In-hospital mortality occurred in 11 patients (8.3\%). Overall survival at 1, 5 and 10 years were, respectively, 86.4\%, $69.7 \%$ and $45.9 \%$. At 10 years, overall survival (group F $53.1 \%$, group R 40.0\%, P=0.330) and freedom from moderate to severe MR (group F 53.1\%, group R 53.8\%, P=0.725) did not differ significantly. Freedom from hospitalization for heart failure was $59.3 \%$. Left ventricular reverse remodelling, defined as a reduction of left ventricular end-systolic volume index $>15 \%$, occurred more commonly in Group R (51.1\%) compared to Group F (23.1\%), P=0.003.

Conclusions: Restrictive mitral annuloplasty was associated with an operative mortality of $8.3 \%$. Heart failure symptoms and significant MR recur in approximately $40 \%$ of patients after 10 years. Survival remained suboptimal and was not influenced by the type of annuloplasty ring.

Keywords: Ischaemic mitral regurgitation; restrictive mitral annuloplasty; coronary artery disease

Submitted Jul 11, 2019. Accepted for publication Nov 12, 2019.

doi: $10.21037 /$ jtd.2019.12.04

View this article at: http://dx.doi.org/10.21037/jtd.2019.12.04

\section{Introduction}

In patients with coronary artery disease, chronic ischaemic mitral regurgitation (CIMR), has a negative prognostic effect on survival $(1,2)$. The primary mechanism of CIMR is ischaemia-induced left ventricular remodeling, causing papillary muscle displacement and apical tenting of the mitral valve (MV) leaflets. The mainstay of surgical correction is performing a restrictive mitral annuloplasty (RMA) with an undersized annuloplasty ring. This aims to eliminate mitral regurgitation (MR) by increasing the 
coaptive leaflet margins (3).

There are various annuloplasty rings available, including flexible, semi-rigid, partial, and complete rings. The effect of each ring type on the long-term results of mitral annuloplasty remains uncertain. We compared the results of concomitant RMA using a flexible or a semi-rigid ring in patients with CIMR who underwent CABG. Endpoints included overall survival, recurrence of $M R$, readmissions for heart failure and New York Heart Association (NYHA) class.

\section{Methods}

This study is a retrospective review of all patients who underwent CABG with concomitant RMA, between January 1999 and December 2015 at our institution. The study was approved by the local institutional review board with a waiver of patient consent (reference: 2013/680/C). The indication for RMA was the presence of MR of moderate or greater degree. Only patients with CIMR whose primary mechanism of MR was leaflet tethering combined with annular dilatation were included in this study. All patients in this study had a history of at least one previous myocardial infarction or a segmental wall motion abnormality seen on echocardiography. Patients who underwent mitral valve replacement, concomitant aortic valve replacement or surgical ventricular restoration were excluded.

\section{Definitions}

Early or operative mortality was defined as mortality within 30 days and/or death before discharge from the index hospitalization. Renal failure was defined as serum creatinine clearance $(\mathrm{CrCl})$ levels lower than $60 \mathrm{~mL} / \mathrm{min}$ as calculated with the Cockroft-Gault formula, or the need for renal replacement therapy. Respiratory failure was defined as the need for mechanical ventilation for more than $24 \mathrm{~h}$ or the need for reintubation. Left ventricular reverse remodeling (LVRR) was defined as a reduction in left ventricular end-systolic volume index (LVESVI) >15\% (4). Pulmonary hypertension was defined as pulmonary arterial systolic pressure greater than $50 \mathrm{mmHg}$.

\section{Surgical technique}

All operations were performed via median sternotomy using moderately hypothermic $\left(30-32{ }^{\circ} \mathrm{C}\right)$ cardiopulmonary bypass instituted with ascending aortic and bicaval cannulation. Aortic cross-clamping, a combination of intermittent blood cardioplegia for myocardial protection and flooding of the surgical field with carbon dioxide was applied in all cases. Intraoperative transesophageal echocardiography was performed routinely in all patients after the induction of anaesthesia to evaluate valvular and ventricular function during surgery. Coronary bypass grafting was performed to all major territories provided that the coronary arteries were not too small $(<1.5 \mathrm{~mm})$ or too heavily calcified. Rings were downsized in standard fashion by choosing a ring at least 2 sizes smaller than the measured height of the anterior leaflet. The model of annuloplasty ring was at the surgeon's discretion. The same group of surgeons performed the surgeries during the whole study period. All surgeons used flexible annuloplasty rings [Cosgrove-Edwards (Edwards Lifesciences, Irvine, CA), Duran (Medtronic, Minneapolis, MN), and St Jude Tailor (St. Jude Medical, St. Paul, Minnesota)] until Oct 2007 , after which a transition of institutional practice towards the use of semi-rigid annuloplasty rings [Physio, Physio II (Edwards Lifesciences, Irvine, CA), St Jude Saddle (St. Jude Medical, St. Paul, Minnesota) and CG Future (Medtronic, Minneapolis, MN)] was adopted. All surgeons used semi-rigid rings after Oct 2007.

Surgical techniques remained largely unchanged during the study period. No patients required reinstitution of cardiopulmonary bypass for correction of greater than mild residual $M R$.

\section{Echocardiography}

All patients underwent a preoperative transthoracic echocardiography. The severity of MR was assessed by experienced cardiologists, integrating quantitative and supporting echocardiographic parameters, according to existing recommendations $(5,6)$. Left ventricular function was evaluated by measuring the ejection fraction and the endsystolic volume (LVESV) according to the modified biplane Simpson's method. Pulmonary artery systolic pressure was extrapolated from Doppler study of the tricuspid flow.

\section{Data and follow-up}

Patient data was collected from case-notes and electronic medical records during each hospitalization. Collected data included baseline demographics, perioperative clinical variables and echocardiographic data. Data regarding follow-up was obtained by direct assessment during scheduled clinic reviews at our institution. 
Table 1 Preoperative clinical data

\begin{tabular}{|c|c|c|c|c|}
\hline Variable & All patients $(n=133)$ & Group F $(n=39)$ & Group R $(n=94)$ & $P$ value \\
\hline Age (years) & $61.9 \pm 9.2$ & $62.4 \pm 10.2$ & $61.7 \pm 8.7$ & 0.796 \\
\hline Gender (male), n (\%) & $103(77.4)$ & $29(74.4)$ & $74(78.7)$ & 0.584 \\
\hline $\operatorname{BSA}\left(m^{2}\right)$ & $1.66 \pm 0.14$ & $1.66 \pm 0.15$ & $1.66 \pm 0.14$ & 0.708 \\
\hline Diabetes mellitus & $66(49.6)$ & $15(38.5)$ & $51(54.3)$ & 0.097 \\
\hline Renal failure $(\mathrm{CrCl}<60 \mathrm{~mL} / \mathrm{min})$ & $23(17.3)$ & $7(17.9)$ & $16(17.0)$ & 0.898 \\
\hline Hypertension & $83(62.4)$ & $21(53.8)$ & $62(66.0)$ & 0.189 \\
\hline Hyperlipidaemia & $84(63.2)$ & $24(61.5)$ & $60(63.8)$ & 0.803 \\
\hline Atrial fibrillation & $12(9.0)$ & 4 (10.3) & $8(8.5)$ & 0.749 \\
\hline COPD & $5(3.8)$ & $1(2.6)$ & $4(4.3)$ & 0.641 \\
\hline NYHA class III or IV & $104(78.2)$ & $31(79.5)$ & $73(77.7)$ & 0.816 \\
\hline
\end{tabular}

Values for continuous variables are expressed as mean \pm standard deviation. BSA, body surface area; COPD, chronic obstructive pulmonary disease; $\mathrm{CrCl}$, creatinine clearance; LVEF, left ventricular ejection fraction; NYHA, New York Heart Association.

\section{Statistical analysis}

Statistical analyses were performed with the Statistical Package for Social Science, version 17 (SPSS, Chicago, IL, USA). Continuous variables were expressed as means with standard deviation. These were compared using a two-tailed $t$-test. Categorical variables, expressed as percentages, were analyzed with $\chi^{2}$ or Fisher's exact test. Preoperative and postoperative echocardiographic parameters were compared using a paired sample t-test. Survival function and freedom from morbid events were presented using Kaplan-Meier survival curves and comparisons performed with log-rank test. Cox multivariable regression analysis was used to identify the independent predictors of long-term outcomes. Preoperative and operative variables with a univariate $\mathrm{P}<0.10$ or those judged to be clinically important were entered into the multivariate Cox model. All two-tailed P values $<0.05$ were taken as significant.

\section{Results}

Between 1999 and 2015, 133 consecutive patients with CIMR underwent CABG with concomitant RMA at our institution. Thirty-nine patients $(29.3 \%)$ received a flexible annuloplasty ring (Group F) and 94 (70.7\%) had a semirigid ring implanted (Group R). A variety of rings were used within each group. In Group F, 33 patients (84.6\%) received a Cosgrove-Edwards ring, 3 (7.7\%) received a Duran ring, and $3(7.7 \%)$ had a St. Jude Medical Tailor ring implanted. In Group R, 64 (68.1\%) received a Physio or Physio II ring, $27(28.7 \%)$ received a CG Future ring, and 3 (3.2\%) had a St. Jude Medical Saddle implanted. Sixteen patients (12.0\%) underwent concomitant tricuspid valve annuloplasty with a rigid MC3 ring (Edwards Lifesciences, Irvine, CA).

Preoperative clinical data are shown in Table 1. There were no significant differences in baseline characteristics between both groups. At presentation, 29 patients (21.8\%) were in NYHA class II, 59 (44.4\%) in class III, and 45 $(33.8 \%)$ in class IV. Preoperative echocardiographic data were similar between the two groups (Table 2). Surgical data are presented in Table 3. All patients underwent CABG, with a mean of $3.3 \pm 0.8$ grafts. Compared to Group F, a larger proportion of patients in Group $\mathrm{R}$ underwent concomitant tricuspid annuloplasty. Intraoperative transoesophageal echocardiogram showed mild (1+) or less MR after MV repair in all patients.

Postoperative complications are shown in Table 4. Complication rates were similar between Group F and 
Table 2 Preoperative echocardiographic data

\begin{tabular}{|c|c|c|c|c|}
\hline Variable & All patients $(n=133)$ & Group F $(n=39)$ & Group R (n=94) & $P$ value \\
\hline Severe, n (\%) & $82(61.7)$ & $28(71.8)$ & $54(57.4)$ & 0.121 \\
\hline Moderate, n (\%) & $51(38.3)$ & $11(28.2)$ & $40(42.6)$ & 0.121 \\
\hline LVEF (\%) & $28.8 \pm 10.2$ & $31.3 \pm 10.9$ & $27.8 \pm 9.5$ & 0.067 \\
\hline LVESD (mm) & $49.4 \pm 9.0$ & $47.8 \pm 10.4$ & $49.9 \pm 8.5$ & 0.295 \\
\hline LVESVI (mL/m²) & $73.9 \pm 32.7$ & $70.7 \pm 32.4$ & $75.0 \pm 32.9$ & 0.537 \\
\hline LVEDD (mm) & $60.8 \pm 7.4$ & $59.7 \pm 8.0$ & $61.2 \pm 7.2$ & 0.357 \\
\hline PASP & $51.4 \pm 15.8$ & $52.7 \pm 13.8$ & $51.1 \pm 16.3$ & 0.690 \\
\hline
\end{tabular}

Values for continuous variables are expressed as mean \pm standard deviation. LAESD, left atrial end-systolic diameter; LVEDD, left ventricular end-diastolic diameter; LVEF, left ventricular ejection fraction; LVESD, left ventricular end-systolic diameter; LVESVI, left ventricular end-systolic volume index; MR, mitral regurgitation; PASP, pulmonary artery systolic pressure.

Table 3 Operative data

\begin{tabular}{|c|c|c|c|c|}
\hline Variable & All patients $(n=133)$ & Group $F(n=39)$ & Group R ( $n=94)$ & $P$ value \\
\hline CPB time (min) & $156 \pm 28$ & $155 \pm 26$ & $156 \pm 29$ & 0.839 \\
\hline AXC time (min) & $96 \pm 22$ & $91 \pm 21$ & $97 \pm 22$ & 0.136 \\
\hline Number of bypass grafts & $3.3 \pm 0.8$ & $3.2 \pm 0.7$ & $3.3 \pm 0.8$ & 0.702 \\
\hline Intraoperative IABP & $54(40.6)$ & $17(43.6)$ & $37(39.4)$ & 0.651 \\
\hline Tricuspid valve annuloplasty & $16(12.0)$ & $1(2.6)$ & $15(16.0)$ & 0.031 \\
\hline
\end{tabular}

Values for continuous variables are expressed as mean \pm standard deviation. AXC, aortic cross clamp; CPB, cardiopulmonary bypass; IABP, intra-aortic balloon pump.

Group R, except for acute renal failure which was more common in Group $\mathrm{R}(\mathrm{P}=0.016)$. In-hospital mortality occurred in 11 patients $(8.3 \%), 4(10.3 \%)$ in the flexible ring group and $7(7.4 \%)$ in the semi-rigid ring group $(\mathrm{P}=0.592)$. Seven patients $(5.3 \%)$ died from multi-organ failure and $4(3.0 \%)$ died from sepsis. All surviving patients were discharged from hospital with mild or less MR. Clinical follow-up and late echocardiographic data were $100 \%$ complete for the 122 patients who survived to hospital discharge. The mean follow-up period was $6.7 \pm 4.0$ years (Group F: $8.7 \pm 4.1$ years vs. Group R: $5.7 \pm 3.6$ years, $\mathrm{P}<0.001)$. The mean echocardiographic follow-up period was 6.0 \pm 3.6 years (Group F: 7.6 \pm 3.8 years vs. Group R: $5.1 \pm 3.3$ years,
$\mathrm{P}=0.007)$.

\section{Late survival}

Fifty-six patients (42.1\%) died during the follow-up period, including in-hospital deaths. Within this group, 26 patients (46.4\%) died of acute myocardial infarction or congestive cardiac failure. The Kaplan-Meier survival curve for all patients is shown in Figure 1. The degree of preoperative MR [moderate $v s$. severe (Figure 2)] did not affect long-term survival $(\mathrm{P}=0.937)$. Comparing Groups $\mathrm{F}$ and $\mathrm{R}$ (Figure 3), overall survival at 5 years was (Group F, $71.3 \% \pm 7.3 \%$; Group R, $69.0 \% \pm 5.5 \%$ ) and at 10 years (Group F, 
Table 4 Postoperative complications

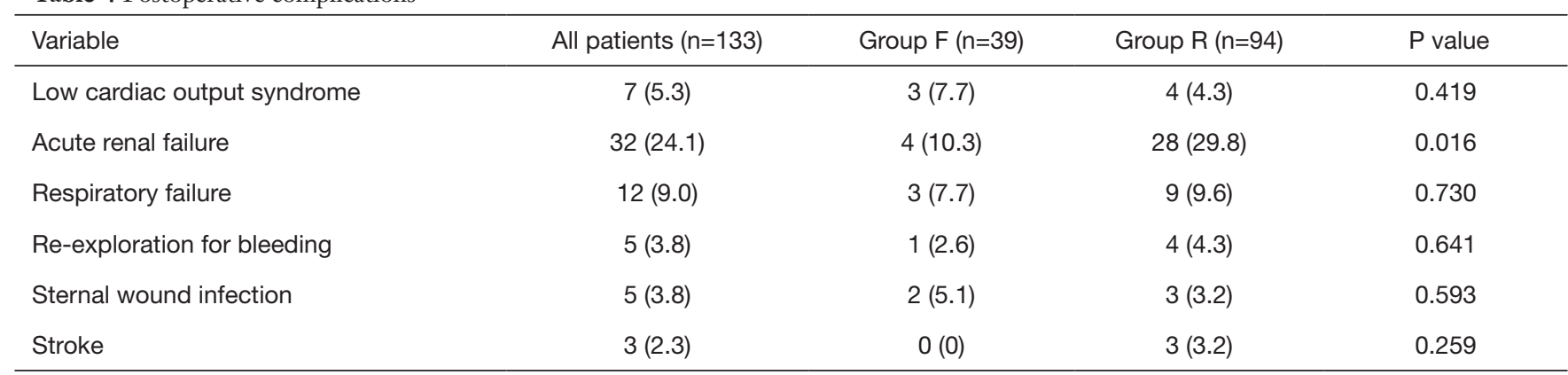

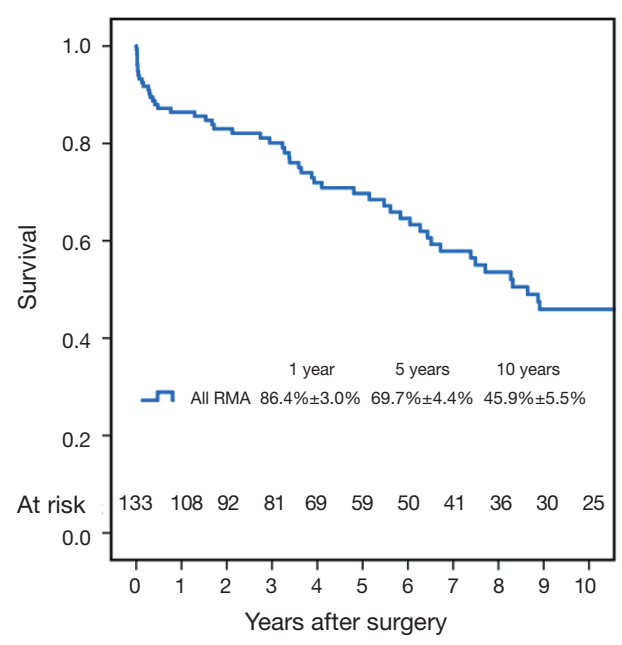

Figure 1 Survival after CABG and RMA. CABG, coronary artery bypass grafting; RMA, restrictive mitral annuloplasty.

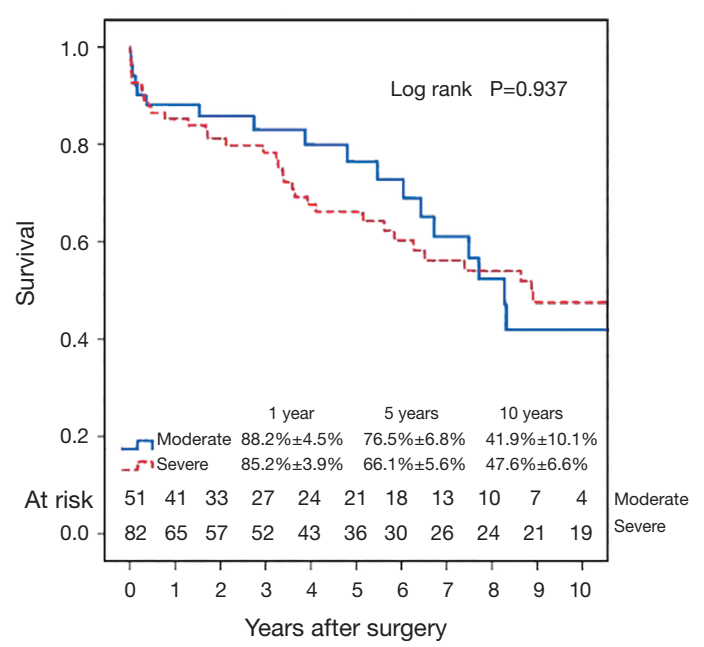

Figure 2 Survival after CABG and RMA, stratified for the degree of preoperative MR. CABG, coronary artery bypass grafting; RMA, restrictive mitral annuloplasty; MR, mitral regurgitation.

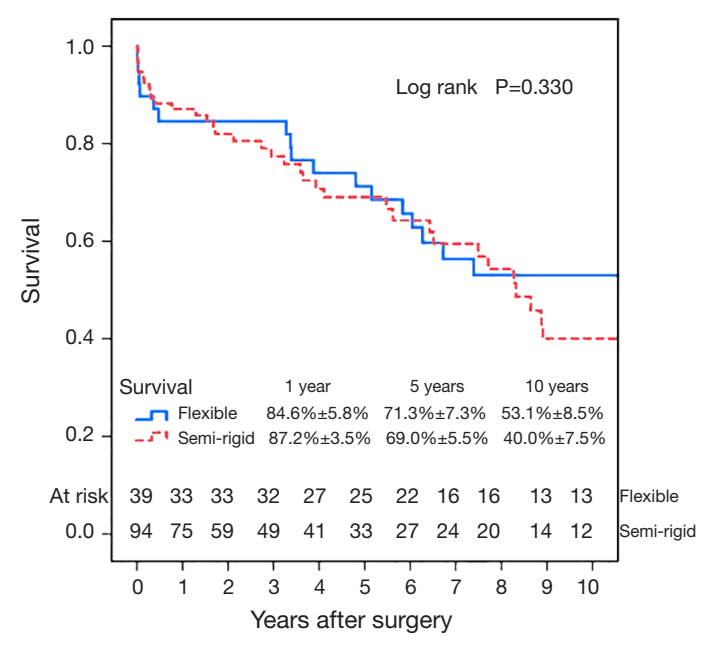

Figure 3 Survival after CABG and RMA, stratified for the type of mitral ring. CABG, coronary artery bypass grafting; RMA, restrictive mitral annuloplasty.

$53.1 \% \pm 8.5 \%$; Group R, $40.0 \% \pm 7.5 \%$ ). There were no statistical differences in the survival rates between Group $\mathrm{F}$ and Group $\mathrm{R}(\mathrm{P}=0.330)$.

Multivariable Cox regression analyses showed that older age $[\mathrm{P}=0.001$; hazard ratio $(\mathrm{HR})=1.08$ per year; $95 \%$ confidence interval (CI), 1.03-1.13] and the presence of preoperative atrial fibrillation $(\mathrm{P}=0.004 ; \mathrm{HR}=4.05 ; 95 \%$ CI, 1.56-10.50) were associated with decreased longterm survival. Variables analyzed included the severity of preoperative MR (moderate/severe), age, type of annuloplasty ring (semi-rigid/flexible), gender, preoperative LVEF, preoperative atrial fibrillation and preoperative LV end-systolic diameter.

\section{Late echocardiographic follow-up}

Echocardiography was performed every 1 to 2 years 
Table 5 Latest echocardiographic data

\begin{tabular}{|c|c|c|c|c|}
\hline Variable & All patients $(n=133)$ & Group F $(n=39)$ & Group R $(n=94)$ & $P$ value \\
\hline LAESD (mm) & $44.3 \pm 7.3^{\star}$ & $46.0 \pm 6.5$ & $43.4 \pm 7.6^{*}$ & 0.071 \\
\hline LVESD (mm) & $46.5 \pm 10.9^{*}$ & $47.6 \pm 10.8$ & $45.9 \pm 10.9^{\star}$ & 0.369 \\
\hline LVESVI (mL/m²) & $63.2 \pm 32.6^{*}$ & $68.3 \pm 29.7$ & $61.3 \pm 33.5^{\star}$ & 0.284 \\
\hline MPG (mmHg) & $4.0 \pm 1.8$ & $3.5 \pm 1.9$ & $4.1 \pm 1.7$ & 0.118 \\
\hline LVEF (\%) & $32.6 \pm 13.2^{*}$ & $33.8 \pm 14.5$ & $32.1 \pm 12.7^{\star}$ & 0.524 \\
\hline PASP & $44.0 \pm 17.3^{*}$ & $51.4 \pm 18.8$ & $41.0 \pm 15.8^{*}$ & 0.012 \\
\hline PASP $>50 \mathrm{mmHg}$ & $40(30.1)$ & $18(46.2)$ & $22(23.4)$ & 0.042 \\
\hline
\end{tabular}

Values for continuous variables are expressed as mean \pm standard deviation. * $\mathrm{P}<0.05$ versus preoperative data. LAESD, left atrial endsystolic diameter; LVEDD, left ventricular end-diastolic diameter; LVEF, left ventricular ejection fraction; LVESD, left ventricular end-systolic diameter; LVESVI, left ventricular end-systolic volume index; LVRR, left ventricular reverse remodeling; MPG, mean pressure gradient; MR, mitral regurgitation; PASP, pulmonary artery systolic pressure.

for patients with mild or less MR and 6-monthly for patients with recurrent moderate or greater MR. The latest postoperative echocardiographic data are shown in Table 5. Overall, the severity of MR was significantly reduced from $2.99 \pm 0.88$ at baseline to $1.10 \pm 0.85$ at latest follow-up. This improvement was evident in both groups. Compared to baseline, the left ventricular dimensions, left atrial dimensions, left ventricular ejection fraction and pulmonary artery systolic pressures improved significantly only in patients from Group R. Compared to Group F, postoperative pulmonary artery systolic pressures were significantly lower in Group $\mathrm{R}(\mathrm{P}=0.012)$.

Left ventricular reverse remodeling occurred in $51.1 \%$ of patients in the semi-rigid ring group compared to $23.1 \%$ in the flexible ring group $(\mathrm{P}=0.003)$. Thirty-two patients (24.1\%) had moderate or worse recurrent MR during follow-up. Figure 4 shows the Kaplan-Meier estimate for recurrent moderate to severe MR in all patients. The severity of preoperative MR was not a predictor of recurrent moderate to severe MR (Figure 5). Comparing Groups $\mathrm{F}$ and $\mathrm{R}$ (Figure 6) at 5 years, freedom from recurrent moderate to severe MR was (Group F, 73.6\% $\pm .6 \%$; Group $\mathrm{R}, 65.2 \% \pm 8.1 \%$ ), and at 10 years (Group F, $53.1 \% \pm 11.5 \%$; Group R, 53.8\% $\pm 9.9 \%)$. There were no statistical differences in the recurrence rates of MR between Group F and Group R ( $\mathrm{P}=0.725)$.

Multivariable Cox regression analysis showed that an increased left ventricular end-systolic diameter (LVESD) was associated with an increased recurrence of moderate or worse $(\geq 2+)$ MR $(\mathrm{P}=0.017$; HR $=1.08$ per $\mathrm{mm}$ increase in LVESD; 95\% CI, 1.01-1.14). The type of annuloplasty ring and degree of preoperative MR did not influence the recurrence of MR. Variables analyzed included the severity of preoperative MR (moderate/severe), age, type of annuloplasty ring (semi-rigid/ flexible), gender, preoperative LVEF, preoperative atrial fibrillation and preoperative LV end-systolic diameter. There were no complications of ring dehiscence in either group.

\section{Hospitalization for beart failure and NYHA class}

Figure 7 shows the Kaplan-Meier estimate for freedom from hospitalization for heart failure. Compared to patients with preoperative moderate MR (Figure 8), hospitalization for heart failure occurred more frequently in patients with preoperative severe MR $(\mathrm{P}=0.023)$. Comparing Groups $\mathrm{F}$ and $\mathrm{R}$ (Figure 9), freedom from hospitalization for heart failure at 5 years was (Group F, 76.2\% $\%$ 7.4\%; Group R, $71.8 \% \pm 6.3 \%$ ) and at 10 years (Group F, $67.2 \% \pm 8.9 \%$; Group R, $50.8 \% \pm 10.7 \%)$. There were no statistical differences in the rates of hospitalization for heart failure between Group F and Group R ( $\mathrm{P}=0.345)$. Amongst the 77 survivors at the latest follow-up, $40(51.9 \%)$ were in NYHA class I, $22(28.6 \%)$ in class II, $13(16.9 \%)$ in class III and 2 $(2.6 \%)$ in class IV. 


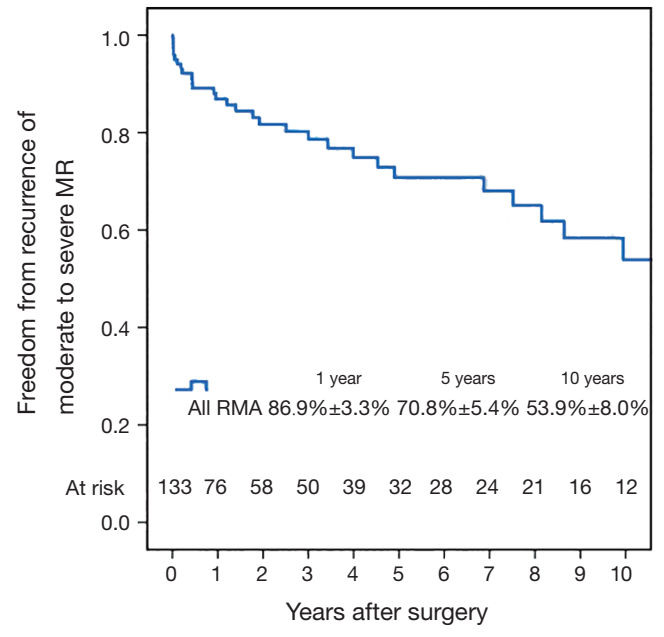

Figure 4 Freedom from recurrence of moderate to severe MR after CABG and RMA. CABG, coronary artery bypass grafting; RMA, restrictive mitral annuloplasty; MR, mitral regurgitation.

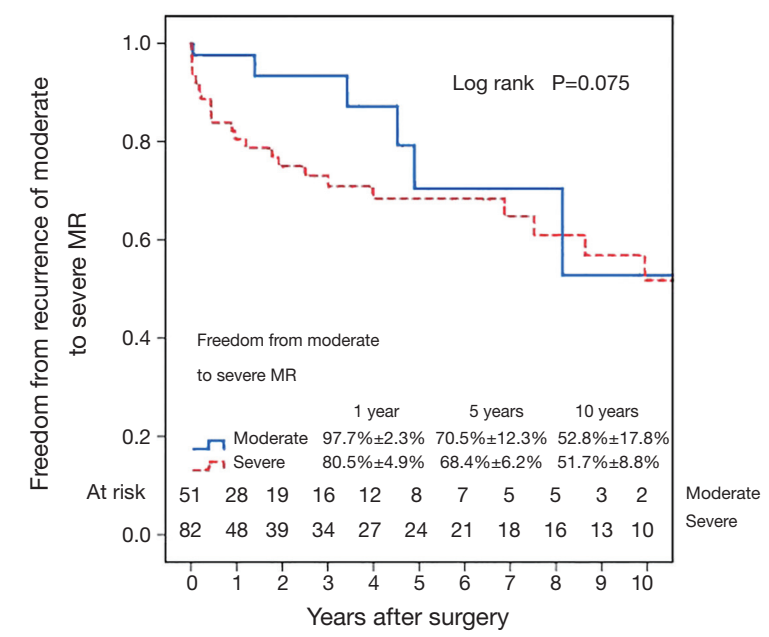

Figure 5 Freedom from recurrence of moderate to severe MR after CABG and RMA, stratified for the degree of preoperative MR. CABG, coronary artery bypass grafting; RMA, restrictive mitral annuloplasty; MR, mitral regurgitation.

\section{Reoperation}

No patients underwent reoperative open heart surgery. One patient $(0.8 \%)$ underwent a percutaneous valve-invalve procedure for recurrent severe MR, 9.1 years after the initial surgery.

\section{Discussion}

This study provides information on early and late clinical

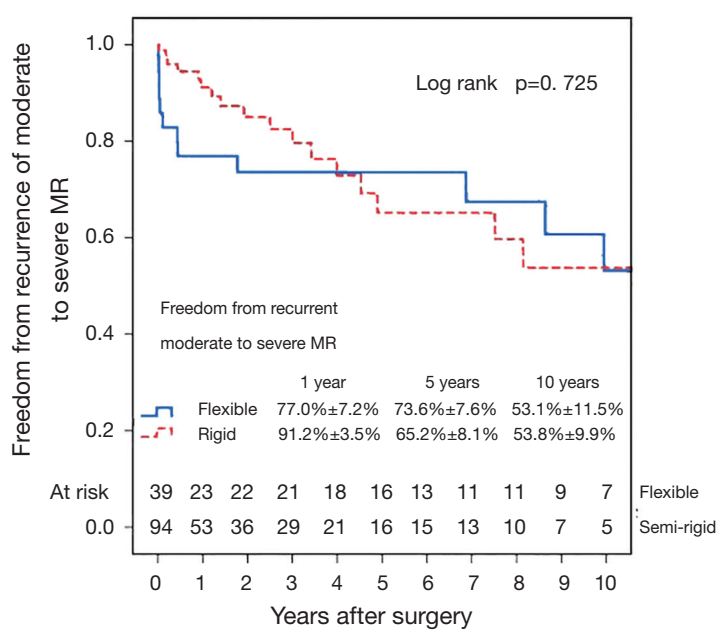

Figure 6 Freedom from recurrence of moderate to severe MR after CABG and RMA, stratified for the type of mitral ring. CABG, coronary artery bypass grafting; RMA, restrictive mitral annuloplasty; MR, mitral regurgitation.

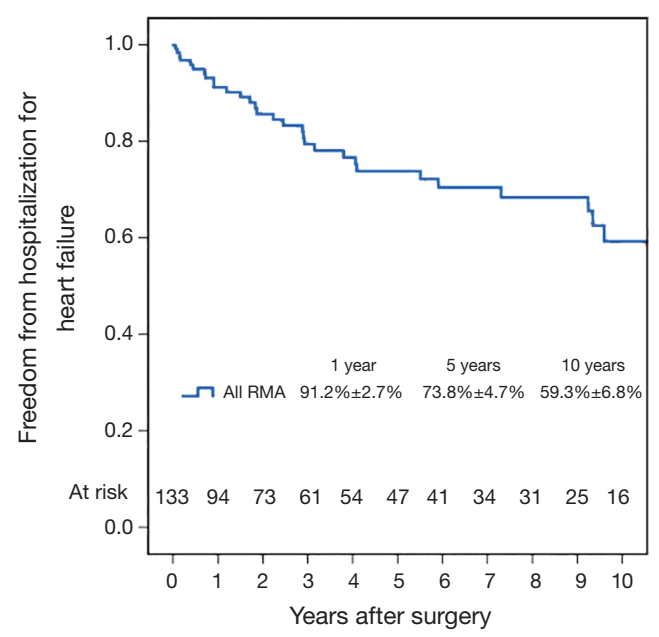

Figure 7 Freedom from hospitalization for heart failure after CABG and RMA. CABG, coronary artery bypass grafting; RMA, restrictive mitral annuloplasty.

outcomes of patients undergoing RMA for CIMR, in a cohort of patients with severely depressed LVEF. More than half $(52.6 \%)$ of these patients had pulmonary hypertension. In contrast to patients with primary MR, the benefit of MV surgery for CIMR remains uncertain. In a small randomized study of 31 patients with moderate CIMR, RMA using a rigid ring was initially effective at reducing the degree of MR but no difference was found in terms of residual $\mathrm{MR}, \mathrm{LV}$ dimensions and function, or clinical outcomes at 


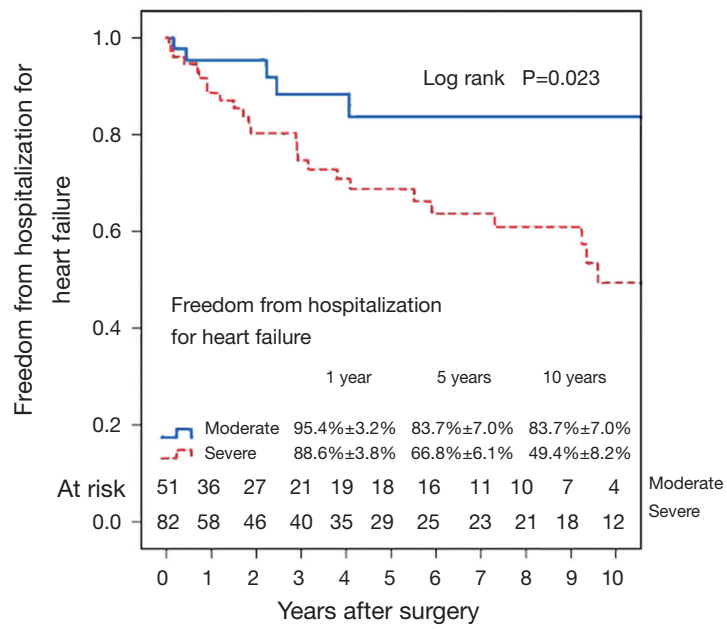

Figure 8 Freedom from hospitalization for heart failure after CABG and RMA, for the degree of preoperative MR. CABG, coronary artery bypass grafting; RMA, restrictive mitral annuloplasty.

1 year when compared to patients who underwent CABG alone (7). Despite its efficacy in reducing MR, a survival advantage of adding MV annuloplasty to CABG has not been demonstrated (8-10).

Our current study cohort consists of patients with severely impaired LVEF undergoing high-risk CABG with RMA. The in-hospital mortality in our cohort $(8.3 \%)$ is comparable with results published previously, ranging from $3.5 \%$ to $10.3 \%(11-17)$.

\section{Long-term survival}

In a cohort of 290 patients with moderate $(52 \%)$ or severe MR (48\%) who underwent CABG with RMA at the Cleveland Clinic, 1-, 5-, and 10-year survival were respectively, 92\%, 74\%, and 39\% (9). Wong et al. reported 1,5 , and 10 -year survival rates of $84 \%, 68 \%$, and $37 \%$ respectively, in a group of 251 patients with moderate ischaemic MR who underwent CABG and RMA (8). In the present study, long-term survival was decreased in patients undergoing CABG \& RMA at an older age and in patients with pre-existing atrial fibrillation. The degree of preoperative MR (moderate or severe) did not affect longterm survival.

In line with results from previous studies, there was no effect of annuloplasty type on survival in our cohort $(11,18,19)$. In contrast, Silberman et al. reported a survival advantage associated with semi-rigid annuloplasty rings,

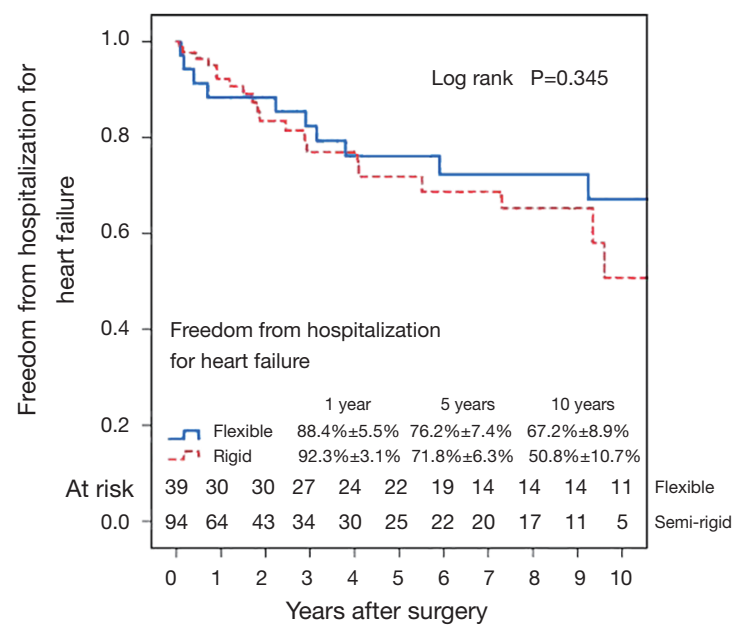

Figure 9 Freedom from hospitalization for heart failure after CABG and RMA, stratified for the type of mitral ring. CABG, coronary artery bypass grafting; RMA, restrictive mitral annuloplasty.

compared to flexible rings (16).

\section{Recurrence of $M R$}

At 5 years, 29\% of our patients developed recurrent moderate or severe MR, increasing to $46 \%$ at 10 years. These rates are comparable to previously published data, ranging from $11.2 \%$ to $32.6 \%(9,10,18,20,21)$. Contrary to other studies which report that significant recurrent MR tends to occur within the first 6 months, the recurrence of MR in our series occurred at a consistent rate throughout the follow-up period $(9,18)$. The use of a non-flexible ring appears to significantly reduce the need for reoperation for recurrent MR (22).

\section{Factors predisposing to recurrence of MR}

Previously described preoperative factors associated with a higher rate of recurrence of MR after annuloplasty include increased preoperative left ventricle dilation with LVEDD $>65 \mathrm{~mm}$ (15), higher severity of preoperative MR $(18,19)$, lower preoperative left ventricular ejection fraction $(18,19)$, anterior leaflet tethering (4), and presence of a basal aneurysm or dyskinesis (23). Recurrent MR is also more frequent with use of partial bands or flexible complete rings, although recurrence rates remain high even with complete rigid rings $(10,11,18,20,22)$.

In the present study, the preoperative LVESD was the 
only predictor of recurrent moderate to severe MR. The type of annuloplasty ring and degree of preoperative MR did not influence the recurrence of MR. Re-intervention rates for recurrent moderate or greater MR were very low, as patients either declined or were assessed to be unfit for high-risk reoperative surgery.

\section{Reverse remodelling}

Despite aggressive downsizing during RMA, no patients experienced functional mitral stenosis. In the present study, a reduction in the degree of MR was observed with both ring types. However, the effect was more pronounced in patients who received semi-rigid rings. Left ventricular reverse remodeling occurred more frequently in patients who received a semi-rigid annuloplasty ring. This may be explained by semi-rigid rings potentially being able to dictate annular shape, stabilize the annular diameter and maintain a fixed inter-trigonal and septal-lateral distance. Despite LVRR occurring more frequently in patients receiving a semi-rigid ring, long-term survival and recurrence of moderate or severe MR did not differ significantly from patients receiving a flexible ring. Braun and associates (24) found that preoperative LVEDD of $65 \mathrm{~mm}$ or less or LVESD of $51 \mathrm{~mm}$ or less were predictive for LVRR, and this conferred a survival advantage (15).

\section{NYHA class}

At 5 years, $26 \%$ of our cohort required readmission for heart failure, with this figure rising to $41 \%$ at 10 years. In a previous report by Mihaljevic and associates, $23 \%$ of patients who underwent CABG + mitral annuloplasty were in NYHA functional class III/IV at 5 years (9). In our series, patients with preoperative severe MR were more likely to be readmitted for heart failure. The Cardiothoracic Surgical Trials Network (CTSN) has provided valuable randomized data from their respective studies on moderate and severe CIMR $(25,26)$. In patients with moderate CIMR, the 2-year rate of moderate or severe residual $M R$ was higher in the CABG-alone group than in the CABG + RMA group $(32.3 \%$ vs. $11.2 \%, \mathrm{P}<0.001)$. Restrictive mitral annuloplasty provided a more durable correction of MR but did not significantly improve survival or reduce overall adverse events or readmissions (25). In patients undergoing MV repair (RMA) or replacement for severe CIMR, there were no significant differences in LVRR or survival at 2 years. Mitral regurgitation recurred more frequently in the repair group (58.8\% vs. $3.8 \%, \mathrm{P}<0.001)$, resulting in more heart-failurerelated adverse events and cardiovascular admissions (26). A meta-analysis of 22 studies (3,815 patients with CIMR) demonstrated significantly reduced perioperative mortality and late mortality following MV repair, compared to MV replacement. Recurrence of at least moderate MR was higher following MV repair (RR, 5.21; 95\% CI, 2.66-10.22; $\mathrm{P}<0.001)(27)$.

\section{Alternative approaches}

In attempts to improve the durability of RMA, 3-dimensional rings have been developed. Using a porcine model, Bouma et al. proposed that the use of undersized saddle-shaped annuloplasty rings (Medtronic Profile 3D (Medtronic, Minneapolis, MN)] in MV repair for CIMR improves leaflet coaptation, which may improve repair durability (28).

Campisi et al. reported impressive results from a study of 157 patients undergoing RMA with the ETlogix ring (Edwards Lifesciences, Irvine, Calif). This annuloplasty ring has a 3-dimensional design for targeted correction of the asymmetric deformation of $\mathrm{P} 2-\mathrm{P} 3$ segments in CIMR. At a median echocardiographic follow-up of 28 months, freedom from $\geq 2+M R$ was $96.6 \%$ (14).

Mitral annuloplasty in CIMR does not prevent coronary artery disease from progressing. By reducing the septolateral dimension of the mitral annulus and increasing leaflet coaptation, RMA only tackles one aspect of the problem related to CIMR. To improve this, subvalvular techniques specifically targeting malpositioned papillary muscles have been described. These techniques have shown promising results (29-31).

\section{Limitations}

This is a retrospective observational study with inherent biases in data collection. Due to the small sample size, statistical analyses may have been underpowered. Being conducted at a single centre, the surgical technique and postoperative care adopted were standardized. The same group of surgeons performed the surgeries throughout the study period. However, our results may not be generalizable to all centres. Viability studies were not conducted routinely and hence unavailable for analysis. Also, advanced echocardiographic measurements such as coaptation height, tenting area and tethering height were not recorded 
routinely in the earlier years of this study and were not analyzed. There was also a temporal difference in the usage of the two classes of rings.

\section{Conclusions}

In this study of 133 patients with CIMR, CABG and RMA with either a flexible or semi-rigid ring, reduced MR and relieved symptoms. Operative mortality was $8.3 \%$. Despite LVRR occurring more frequently in patients receiving a semi-rigid ring, survival and recurrence of significant $M R$ were not influenced by the type of ring used. Survival and durability of MV repair in patients with CIMR remain suboptimal, with heart failure symptoms and significant MR recurring in approximately $40 \%$ of patients after 10 years. Adjunct subvalvular techniques may play a role in improving outcomes.

\section{Acknowledgments}

The authors thank Clara Zhang and Selena Chew for their editorial assistance.

\section{Footnote}

Conflicts of Interest: The authors have no conflicts of interest to declare.

Ethical Statement: The authors are accountable for all aspects of the work in ensuring that questions related to the accuracy or integrity of any part of the work are appropriately investigated and resolved. The study was approved by the local institutional review board with a waiver of patient consent (reference: 2013/680/C).

\section{References}

1. Grigioni F, Enriquez-Sarano M, Zehr KJ, et al. Ischemic mitral regurgitation: long-term outcome and prognostic implications with quantitative Doppler assessment. Circulation 2001;103:1759-64.

2. Rossi A, Dini FL, Faggiano P, et al. Independent prognostic value of functional mitral regurgitation in patients with heart failure. A quantitative analysis of 1256 patients with ischaemic and nonischaemic dilated cardiomyopathy. Heart 2011;97:1675-80.

3. Bolling SF, Deeb GM, Brunsting LA, et al. Early outcome of mitral valve reconstruction in patients with end-stage cardiomyopathy. J Thorac Cardiovasc Surg 1995;109:676-82 .

4. Gelsomino $S$, van Garsse L, Lucà $F$, et al. Impact of preoperative anterior leaflet tethering on the recurrence of ischemic mitral regurgitation and the lack of left ventricular reverse remodeling after restrictive annuloplasty. J Am Soc Echocardiogr 2011;24:1365-75.

5. Zoghbi WA, Enriquez-Sarano M, Foster E, et al. Recommendations for evaluation of the severity of native valvular regurgitation with two-dimensional and Doppler echocardiography. J Am Soc Echocardiogr 2003;16:777-802.

6. Kron IL, LaPar DJ, Acker MA, et al. 2016 update to The American Association for Thoracic Surgery consensus guidelines: Ischemic mitral valve regurgitation. J Thorac Cardiovasc Surg 2017;153:1076-9.

7. Bouchard D, Jensen H, Carrier M, et al. Effect of systematic downsizing rigid ring annuloplasty in patients with moderate ischemic mitral regurgitation. J Thorac Cardiovasc Surg 2014;147:1471-7.

8. Wong DR, Agnihotri AK, Hung JW, et al. Long-term survival after surgical revascularization for moderate ischemic mitral regurgitation. Ann Thorac Surg 2005;80:570-7.

9. Mihaljevic T, Lam BK, Rajeswaran J, et al. Impact of mitral valve annuloplasty combined with revascularization in patients with functional ischemic mitral regurgitation. J Am Coll Cardiol 2007;49:2191-201.

10. Acker MA, Parides MK, Perrault LP, et al. Mitral-valve repair versus replacement for severe ischemic mitral regurgitation. N Engl J Med 2014;370:23-32.

11. Kwon MH, Lee LS, Cevasco M, et al. Recurrence of mitral regurgitation after partial versus complete mitral valve ring annuloplasty for functional mitral regurgitation. J Thorac Cardiovasc Surg 2013;146:616-22.

12. Grossi EA, Woo YJ, Patel N, et al. Outcomes of coronary artery bypass grafting and reduction annuloplasty for functional ischemic mitral regurgitation: a prospective multicenter study (Randomized Evaluation of a Surgical Treatment for Off-Pump Repair of the Mitral Valve). J Thorac Cardiovasc Surg 2011;141:91-7.

13. Bax JJ, Braun J, Somer ST, et al. Restrictive annuloplasty and coronary revascularization in ischemic mitral regurgitation results in reverse left ventricular remodeling. Circulation 2004;110:II103-8.

14. Campisi S, Fuzellier JF, Haber B, et al. Mid-term results of mitral valve repair for ischemic mitral regurgitation with ETlogix ring: A single-center study. Int J Cardiol 2016;222:924-30.

15. Braun J, van de Veire NR, Klautz RJ, et al. Restrictive mitral 
annuloplasty cures ischemic mitral regurgitation and heart failure. Ann Thorac Surg. 2008;85:430-6; discussion 436-7.

16. Silberman S, Klutstein MW, Sabag T, et al. Repair of ischemic mitral regurgitation: comparison between flexible and rigid annuloplasty rings. Ann Thorac Surg 2009;87:1721-6.

17. Crabtree TD, Bailey MS, Moon MR, et al. Recurrent mitral regurgitation and risk factors for early and late mortality after mitral valve repair for functional ischemic mitral regurgitation. Ann Thorac Surg 2008;85:1537-42.

18. McGee EC, Gillinov AM, Blackstone EH, et al. Recurrent mitral regurgitation after annuloplasty for functional ischemic mitral regurgitation. J Thorac Cardiovasc Surg 2004;128:916-24.

19. von Oppell UO, Stemmet F, Brink J, et al. Ischemic mitral valve repair surgery. J Heart Valve Dis 2000;9:64-73.

20. Smith PK, Puskas JD, Ascheim DD, et al. Surgical treatment of moderate ischemic mitral regurgitation. $\mathrm{N}$ Engl J Med 2014;371:2178-88.

21. Hung J, Papakostas L, Tahta SA, et al. Mechanism of recurrent ischemic mitral regurgitation after annuloplasty: continued LV remodeling as a moving target. Circulation 2004;110:II85-90.

22. Spoor MT, Geltz A, Bolling SF. Flexible versus nonflexible mitral valve rings for congestive heart failure: differential durability of repair. Circulation 2006;114:I67-71.

23. Kron IL, Hung J, Overbey JR, et al. Predicting recurrent mitral regurgitation after mitral valve repair for severe ischemic mitral regurgitation. J Thorac Cardiovasc Surg

Cite this article as: Pang PYK, Huang MJ, Tan TE, Lim SL, Naik MJ, Chao VTT, Sin YK, Lim CH, Chua YL. Restrictive mitral valve annuloplasty for chronic ischaemic mitral regurgitation: outcomes of flexible versus semi-rigid rings. J Thorac Dis 2019;11(12):5096-5106. doi: 10.21037/ jtd.2019.12.04 2015;149:752-61.e1.

24. Braun J, Bax JJ, Versteegh MI, et al. Preoperative left ventricular dimensions predict reverse remodeling following restrictive mitral annuloplasty in ischemic mitral regurgitation. Eur J Cardiothorac Surg 2005;27:847-53.

25. Michler RE, Smith PK, Parides MK, et al. Two-Year Outcomes of Surgical Treatment of Moderate Ischemic Mitral Regurgitation. N Engl J Med 2016;374:1932-41.

26. Goldstein D, Moskowitz AJ, Gelijns AC, et al. Two-Year Outcomes of Surgical Treatment of Severe Ischemic Mitral Regurgitation. N Engl J Med 2016;374:344-53.

27. Virk SA, Sriravindrarajah A, Dunn D, et al. A meta-analysis of mitral valve repair versus replacement for ischemic mitral regurgitation. Ann Cardiothorac Surg 2015;4:400-10.

28. Bouma W, Aoki C, Vergnat M, et al. Saddle-Shaped Annuloplasty Improves Leaflet Coaptation in Repair for Ischemic Mitral Regurgitation. Ann Thorac Surg 2015;100:1360-6.

29. Kron IL, Green GR, Cope JT. Surgical relocation of the posterior papillary muscle in chronic ischemic mitral regurgitation. Ann Thorac Surg 2002;74:600-1.

30. Fattouch K, Castrovinci S, Murana G, et al. Papillary muscle relocation and mitral annuloplasty in ischemic mitral valve regurgitation: midterm results. J Thorac Cardiovasc Surg 2014;148:1947-50.

31. Nappi F, Lusini M, Spadaccio C, et al. Papillary Muscle Approximation Versus Restrictive Annuloplasty Alone for Severe Ischemic Mitral Regurgitation. J Am Coll Cardiol 2016;67:2334-46. 